\title{
Improvement of liver function by the administration of oyster extract as a dietary supplement to habitual alcohol drinkers: A pilot study
}

\author{
KENJI OSAKI $^{1}$, YOSHIO SHIMIZU ${ }^{2}$, TETSURO YAMAMOTO ${ }^{3}$, FUMIHARU MIYAKE ${ }^{4}$, \\ SUMIO KONDO $^{5}$ and HIDEYO YAMAGUCHI ${ }^{3}$
}

\author{
${ }^{1}$ Tokyo Branch, Bizen Chemical Co. Ltd., Tokyo 103-0023; ${ }^{2}$ Okayama Head Office, Bizen Chemical Co. Ltd., \\ Okayama 709-0716; ${ }^{3}$ Research Center, TTC Co. Ltd., Tokyo 113-0021; ${ }^{4}$ Product Formulation Division, \\ Bizen Chemical Co. Ltd., Okayama 709-0716; ${ }^{5}$ Medical Corporation Kenshokai, \\ Fukushima Healthcare Center, Osaka 553-0004, Japan
}

Received July 1, 2014; Accepted April 28, 2015

DOI: $10.3892 / \mathrm{etm} .2015 .2563$

\begin{abstract}
Alcoholic liver disease (ALD) is characterized by elevated serum $\gamma$-glutamyltransferase (GGT) activity with hepatic steatosis, hepatitis or occasionally fibrosis that may progress to cirrhosis. The potential therapeutic role of oyster extract $(\mathrm{OE})$ or OE-containing dietary supplements (OE supplement) in ALD has seldom been evaluated. In the present study, 84 adults who had an alcohol-drinking habit and marginally high serum GGT levels were enrolled in a randomized, double-blind, placebo-controlled feeding trial to study the effect on alcohol-impaired liver function as reflected by an increased serum level of GGT, as well as the safety, of an OE supplement. The subjects were randomized to receive either an OE supplement (OE group) or placebo (placebo group). There were 42 subjects ( 31 males and 11 females) in each group, and all the enrolled subjects entered the study. Four individuals (5\%) dropped out for reasons unassociated with the study and 6 other subjects were excluded from the efficacy analysis because they did not maintain the required frequency of alcohol intake. As a result, 38 subjects in the placebo group and 36 in the $\mathrm{OE}$ group underwent efficacy assessment. Assays of GGT and other liver enzymes were performed at baseline (week 0) and at weeks 4, 8 and 12 of the intervention period. The mean serum levels of GGT in the placebo group gradually increased, while those in the OE group tended to decrease, although no significant within-group differences were observed for either group. A significant between-group difference in the change of mean
\end{abstract}

Correspondence to: Mr. Kenji Osaki, Tokyo Branch, Bizen Chemical Co. Ltd., Kdc Nihonbashi Building, 1-5-11 Nihonbashi-Honcho, Tokyo 103-0023, Japan

E-mail:kenji-os@bizen-c.co.jp

Key words: liver function, oyster extract, habitual alcohol drinking, feeding trial, taurine, $\gamma$-glutamyltransferase
GGT from baseline was, however, found at week $12(\mathrm{P}=0.049)$. No OE supplement-associated untoward side-effects nor any abnormal changes in routine laboratory tests and anthropometric parameters were observed throughout the 12-week intervention. An OE supplement shows promise in reducing risk factors associated with ALD in adults with an alcohol intake habit.

\section{Introduction}

Alcohol consumption is customary in the majority of cultures and alcohol abuse is common worldwide. The burden of excessive alcohol consumption-associated disease and mortality remains significant in numerous countries including Asian countries, such as China, Korea and Japan. The World Health Organization reported that, globally, alcohol is responsible for $\sim 4 \%$ of all mortality and alcoholic liver disease (ALD) accounts for one-quarter of all alcohol-attributable fatalities (1). In China, alcohol abuse has been considered the second leading cause of liver disease (2). A similar trend of alcohol consumption-associated burden of disease, in particular ALD, has also been seen in Korea $(3,4)$, as well as in Japan $(5,6)$.

The most prevalent type of ALD is alcoholic fatty liver (or hepatic steatosis) and a significant subset of the individuals with hepatic steatosis progress to severe alcoholic hepatitis, of which the 6-month mortality rate is $\leq 40 \%$ (7). Severe alcoholic hepatitis also places an enormous burden on stretched healthcare resources $(8,9)$. Unfortunately, however, there is no established therapy for ALD, and the treatment has been limited to supportive management and nutritional supplementation without clear improvements in outcome $(10,11)$. There is, therefore, a rationale for preventing habitual alcohol drinkers from developing steatosis, an initially occurring reversible-type of liver injury.

Taurine (2-aminoethanesulfonic acid), a non-metabolizable sulfur-containing $\beta$-amino acid, is a major free intracellular amino acid present in numerous tissues of humans and animals (12). Taurine has been shown to have beneficial 
effects on liver injury in various animal models. For example, taurine supplementation prevents hepatic steatosis and other alcohol-induced liver diseases in rats (13-15) and the development of steatosis in hamsters fed with high-fat/cholesterol diets (16). Despite convincing evidence in animal models, studies examining the metabolic response to supplementation with taurine in the context of alcoholic liver injury or dysfunction in humans are lacking, particularly in Japanese and other Asian nations who have a high proportion of poor alcohol metabolizers due to a genetic deficiency in the enzyme aldehyde dehydrogenase $(17,18)$.

Oyster extract (OE) in a powder form is a nutraceutical that is produced from a species of oyster (Crassostrea gigas) by a biochemical preparation process. In Japan, several dietary supplements containing powdered OE are commercially available and particularly popular among habitual alcohol drinkers who are at a high risk of ALD. The objective of the present human study was to assess the effects of 12 weeks of consumption of OE-containing supplements (OE supplement) on the serum levels of $\gamma$-glutamyltransferase (GGT) in Japanese adults with an alcohol-drinking habit. Serum GGT levels were selected as the major outcome measure as the enzyme is an established biomarker of liver function and chronically heavy alcohol intake (19-21).

\section{Subjects and methods}

Study design and ethics. A randomized, double-blind, placebo-controlled study was designed to assess the efficacy and safety of the OE supplement for improving the alcohol-impaired liver function in enrolled subjects when compared with placebo. The study was performed from May 2013 through September 2013 at the Medical Corporation Kenshokai, Fukushima Healthcare Center (Osaka, Japan). The study protocol was approved by the Ethics Committee of the Fukushima Healthcare Center (approval date, 10 May 2013) and was in accordance with the principles of the Helsinki Declaration of 1995 (as revised in October 2008) and the Ethical Guidelines for Epidemiological Research (enacted in 2004 and revised in 2008 by the Japanese Government). Written informed consent was obtained from all study subjects prior to their enrollment in the study. To ensure privacy, all records were identified with an anonymous subject identification number.

Subjects and eligibility. A total of 84 Japanese adults, aged 20-65 years, consisting of males and females at a ratio of $\sim 7$ to 3 who had an alcohol drinking habit (5-7 times per week), a GGT level between 50 and $150 \mathrm{IU} / 1$ and a body mass index (BMI) between 18 and $30 \mathrm{~kg} / \mathrm{m}^{2}$, were voluntarily recruited and included in this study. Individuals were excluded if they were: i) Currently taking medications, health foods and/or foods for specified health uses that may alter alcohol metabolism or liver enzyme activities; ii) habitually consuming $\mathrm{OE}$; iii) positive for hepatitis virus tests (e.g., hepatitis B surface antigen and anti-hepatitis $\mathrm{C}$ virus antibody); iv) at risk of developing a study-associated allergic reaction, having a history of serious disease that required regular medication; v) with abnormal values of anthropometric, physiological or laboratory test parameters appreciably deviated from the normal range; vi) participating in any other clinical trial at the start time of the present study; vii) pregnant or breastfeeding; and viii) with the presence of any clinically significant medical condition that was judged by the medical investigator to preclude the inclusion of the participant in the study.

Dietary intervention and subject assignment. The OE supplement used in this study was a commercially available product manufactured by Bizen Chemical Co. Ltd. (Okayama, Japan) in the form of a $386 \mathrm{mg}$ tablet containing $333 \mathrm{mg}$ powdered OE, whose major constituents are glycogen $(>50 \% \mathrm{w} / \mathrm{w})$ and taurine $(>5 \% \mathrm{w} / \mathrm{w})$. The tablet also contained a $53 \mathrm{mg}$ vehicle mixture of yeast cell wall, sucrose esters of fatty acids, tricalcium phosphate, maize protein, shellac, glycerin, titanium dioxide and carnauba wax. In the placebo tablet, OE was replaced by maltitol, dextrins and microcrystalline cellulose, in a total amount of $386 \mathrm{mg}$, and the contents were colored to make them similar in appearance to the contents of the $\mathrm{OE}$ supplement tablet.

Throughout the 12-week treatment period, each subject was required to keep a study diary of their allocated tablet intake, alcohol consumption, dietary composition, physical activity as measured by a passometer, all medications or therapies received, and any adverse events experienced. The subjects were also instructed to maintain their usual habits of alcohol drinking, food and beverage intake and current exercise program and to avoid the intake of health foods that may alter liver function.

The study intervention began $\sim 1$ week following the screening of eligible subjects. Male and female subjects were separately and sequentially assigned one of the two types of the masked study tablet (OE supplement or placebo) based on random number tables and were randomized (1:1) to the OE supplement (OE group) and placebo (placebo group). The subjects were required to consume three tablets of either OE supplement $(1,000 \mathrm{mg}$ as $\mathrm{OE}$ and $>50 \mathrm{mg}$ as taurine) or placebo products daily prior to the evening meal with the aid of a cup of water over the 12-week intervention period. Consumption of $<80 \%$ of the prescribed course of the allocated product was considered as non-compliance and non-compliant subjects were excluded from the efficacy assessment.

To assess efficacy and safety, medical inspections, measurements of serum liver enzyme levels and anthropometric and hemodynamic parameters, as well as other laboratory tests, were performed prior to the start of intervention (week 0) and at weeks 4,8 and 12 .

Laboratory tests for serum analysis and urinalysis. A commercial laboratory test company (Mitsubishi Chemical Medience Corporation, Osaka, Japan) analyzed all biochemical and hematological blood and urine parameters including: GGT, alanine transaminase (ALT), aspartate transaminase (AST), low-density lipoprotein cholesterol, high-density lipoprotein cholesterol, triglycerides, total serum protein, albumin, total bilirubin, lactate dehydrogenase, blood urea nitrogen, creatinine, uric acid, sodium, potassium, chloride, blood glucose, white blood cells, red blood cells, hemoglobin, hematocrit and platelets. Urine samples were used for the analysis of 
Table I. Demographic and biochemical data for subjects in the placebo and OE groups at entry.

\begin{tabular}{|c|c|c|c|c|}
\hline Measurement & Normal range & $\begin{array}{l}\text { Placebo group } \\
\qquad(\mathrm{n}=42)\end{array}$ & $\begin{array}{c}\text { OE group } \\
(\mathrm{n}=42)\end{array}$ & $\mathrm{P}$-value \\
\hline Age (years) & & $48.8 \pm 9.1$ & $46.3 \pm 8.7$ & 0.205 \\
\hline Gender (male/female, n) & & $31 / 11$ & $31 / 11$ & 1.000 \\
\hline Height $(\mathrm{cm})$ & & $168 \pm 8$ & $166 \pm 8$ & 0.535 \\
\hline Weight (kg) & & $67.0 \pm 11.9$ & $67.6 \pm 11.0$ & 0.817 \\
\hline BMI $\left(\mathrm{kg} / \mathrm{m}^{2}\right)$ & & $23.8 \pm 2.9$ & $24.3 \pm 3.2$ & 0.378 \\
\hline Systolic BP (mmHg) & & $135 \pm 18$ & $132 \pm 14$ & 0.428 \\
\hline Diastolic BP (mmHg) & & $85 \pm 10$ & $83 \pm 11$ & 0.427 \\
\hline Heart rate (beats/min) & & $74 \pm 10$ & $76 \pm 10$ & 0.386 \\
\hline LDL-cholesterol (mg/dl) & $65-139$ & $124 \pm 38$ & $129 \pm 43$ & 0.570 \\
\hline HDL-cholesterol (mg/dl) & $40-85$ & $65 \pm 18$ & $64 \pm 17$ & 0.684 \\
\hline $\mathrm{TG}(\mathrm{mg} / \mathrm{dl})$ & $30-149$ & $167 \pm 132$ & $157 \pm 104$ & 0.718 \\
\hline GGT (IU/1) & $10-50$ & $78.1 \pm 29.5$ & $78.3 \pm 30.7$ & 0.974 \\
\hline ALT (IU/1) & $5-45$ & $28.0 \pm 15.4$ & $30.4 \pm 14.4$ & 0.475 \\
\hline $\operatorname{AST}(\mathrm{IU} / \mathrm{l})$ & $10-40$ & $25.8 \pm 7.2$ & $26.1 \pm 10.1$ & 0.842 \\
\hline
\end{tabular}

Data are presented as the mean \pm standard deviation with the exception of gender. The P-value refers to the between-group difference assessed by Student's unpaired t-test for all parameters with the exception of gender and by the $\chi^{2}$ test for gender. OE, oyster extract; BMI, body mass index; BP, blood pressure; LDL, low-density lipoprotein; HDL, high-density lipoprotein; TG, triglycerides; GGT, $\gamma$-glutamyltransferase; ALT, alanine transaminase; AST, aspartate transaminase.

qualitative protein, qualitative glucose, qualitative occult blood, urobilinogen and $\mathrm{pH}$.

Anthropometrics and hemodynamics. Height was measured at the beginning of the study. Body weight was measured and BMI calculated at weeks $0,4,8$ and 12. Systolic and diastolic blood pressures (BP) were measured, with subjects in the sitting position following $10 \mathrm{~min}$ rest, using an automatic sphygmomanometer.

Statistical analysis. Data are presented as the mean \pm standard deviation. Only data from subjects completing the study were included in the efficacy analysis. The baseline characteristics of the randomized subjects were compared between the placebo and OE groups by Student's unpaired t-test and the $\chi^{2}$ test for mean values and proportions of parameters, respectively. Between-group comparisons of efficacy results were performed by comparing the changes from baseline (week 0) that were calculated by subtracting the initial (week 0) value from the value at each prescribed assessment time-point (weeks 4, 8 and 12) between the placebo and $\mathrm{OE}$ groups with the use of Student's unpaired t-test. Within-group differences of group means were compared by Student's paired t-test. $\mathrm{P}<0.05$ was considered to indicate a statistically significant difference.

\section{Results}

Characteristics of subjects. Eighty-four individuals were enrolled in the study and assigned to either the placebo or OE group (each $n=42 ; 31$ males and 11 females). Table I shows the baseline data for the randomized subjects expressed as group mean in the placebo and OE groups. At enrollment, there were no significant differences in the values of any anthropometric, hemodynamic and biochemical parameters between the two groups. It is also shown in this table that the mean baseline levels of all parameters including the liver enzymes ALT and AST, with the exception of the liver enzyme GGT, for the two groups were within the normal range or marginally increased above it.

Four of the 84 enrolled subjects [placebo group $(n=2$; 2 males) and OE group ( $n=2 ; 1$ male and 1 female)] dropped out of the study during weeks 1-4 for personal reasons unrelated to the study, and thus 80 subjects completed the study, giving a retention rate of $95 \%$. The ingestion rate of allocated products in all individual subjects was $>86.9 \%$ with the average value being $99.2 \%$. Six subjects were excluded from efficacy analysis for the following reasons: i) $>2$-fold increase in GGT level compared with the baseline ( $\geq 200$ IU/1, probably due to excessive alcohol intake $[n=3 ; 1$ female (placebo group)] and 2 males (OE group)]; ii) insufficient alcohol intake in frequency (3.6 to 4.5 times per week on average) compared with 5-7 times for requirement $[\mathrm{n}=3 ; 1$ female (placebo group) and 2 males (OE group)]. Thus, 38 subjects (29 males and 9 females) in the placebo group and 36 (26 males and 10 females) in the OE group were subjected to efficacy analysis.

Effect on GGT and other liver enzymes. As shown in Table II, the mean baseline (week 0) GGT, ALT and AST activities were not statistically different between the placebo and OE groups. During the 12-week intervention, the GGT activity tended to elevate gradually in the placebo group, being increased by $12 \%$ compared with the activity at week $0(88.5$ versus $79.3 \mathrm{IU} / 1$, respectively), although the change in the activity of the enzyme did not reach statistical significance $(\mathrm{P}=0.077)$. By contrast, GGT levels appeared to be reduced 
Table II. Liver enzyme activity during the 12-week intervention in the placebo $(n=36)$ and OE groups $(n=38)$.

\begin{tabular}{|c|c|c|c|c|c|c|c|}
\hline \multirow[b]{2}{*}{ Enzyme } & \multirow[b]{2}{*}{ Week 0 activity } & \multicolumn{2}{|c|}{ Week 4} & \multicolumn{2}{|c|}{ Week 8} & \multicolumn{2}{|c|}{ Week 12} \\
\hline & & Activity & P-value & Activity & P-value & Activity & P-value \\
\hline \multicolumn{8}{|c|}{ GGT (IU/l) } \\
\hline Placebo & $79.3 \pm 29.5$ & $77.5 \pm 35.7(-2 \%)$ & 0.701 & $80.5 \pm 37.8(2 \%)$ & 0.768 & $88.5 \pm 41.8(12 \%)$ & 0.077 \\
\hline $\mathrm{OE}$ & $76.9 \pm 32.0$ & $71.7 \pm 30.7(-7 \%)$ & 0.278 & $74.5 \pm 28.2(-3 \%)$ & 0.583 & $70.6 \pm 30.5(-8 \%)$ & 0.290 \\
\hline \multicolumn{8}{|l|}{ ALT (IU/l) } \\
\hline Placebo & $28.7 \pm 15.9$ & $27.0 \pm 12.7(-2 \%)$ & 0.388 & $26.5 \pm 12.8(-8 \%)$ & 0.358 & $31.4 \pm 20.2(9 \%)$ & 0.348 \\
\hline OE & $31.0 \pm 15.1$ & $27.2 \pm 13.0(-12 \%)$ & 0.071 & $29.9 \pm 15.6(-4 \%)$ & 0.471 & $27.5 \pm 13.6(-10 \%)$ & 0.058 \\
\hline \multicolumn{8}{|l|}{ AST (IU/l) } \\
\hline Placebo & $25.9 \pm 7.3$ & $24.8 \pm 5.3(-4 \%)$ & 0.310 & $25.4 \pm 6.0(-2 \%)$ & 0.728 & $26.4 \pm 8.0(2 \%)$ & 0.745 \\
\hline $\mathrm{OE}$ & $25.5 \pm 7.7$ & $23.8 \pm 7.2(-7 \%)$ & 0.101 & $25.6 \pm 10.6(0 \%)$ & 0.966 & $22.9 \pm 6.8(-10 \%)$ & 0.168 \\
\hline
\end{tabular}

Activity data are presented as the mean \pm standard deviation. Percentage changes (presented in the brackets) are from week 0 . The statistical significance of a within-group difference in measurement from week 0 was assessed by the Student's paired t-test. OE, oyster extract; GGT, $\gamma$-glutamyltransferase; ALT, alanine transaminase; AST, aspartate transaminase.

Table III. Alcohol intake in subjects of the placebo and OE groups (g/day).

\begin{tabular}{lcccc}
\hline Group & Weeks 1-4 & Weeks 5-8 & Weeks 9-12 & Weeks 1-12 \\
\hline Placebo (n=36) & $54 \pm 29$ & $54 \pm 28$ & $52 \pm 27$ & $53 \pm 27$ \\
OE (n=38) & $47 \pm 24$ & $46 \pm 28$ & $47 \pm 28$ & $47 \pm 25$ \\
P-value & 0.222 & 0.188 & 0.446 & 0.250 \\
\hline
\end{tabular}

Data are presented as the mean \pm standard deviation. The P-value refers to the between-group difference assessed by the Student's unpaired t-test. OE, oyster extract.

throughout the intervention period in the OE group, whose mean value at week 12 was $8 \%$ lower than that at baseline. However, the reduction in values was not statistically significant (70.6 versus $76.9 \mathrm{IU} / 1$, respectively; $\mathrm{P}=0.290$ ).

Fig. 1 shows the comparison of changes from baseline in the mean value of GGT at weeks 4,8 and 12 between the placebo and OE groups. There was a significant difference between the placebo and OE groups at week 12 (mean change from baseline: placebo, 9.2 $\pm 31.1 \mathrm{IU} / 1$; OE, $-6.3 \pm 35.4$ IU/1; $\mathrm{P}<0.05$ ) but not at weeks 4 and 8 .

A similar trend showing an increase and decrease in activity in the placebo and OE groups, respectively, was also seen for ALT and AST; however, there was no significant within-group or between-group differences at any time-point during the intervention period (Table II, Fig. 1).

Alcohol intake analysis. Self-reported intake of alcohol is presented in Table III. No difference was observed in the mean alcohol intake between the placebo and OE groups at any assessment time-point throughout the study period.

Safety and tolerability. The OE supplement, as well as the placebo, used in this study was well tolerated. No subject withdrew due to adverse events. Only 3 of the 42 subjects $(7 \%)$ in the OE group and 12 of the 42 subjects (29\%) in the placebo group reported minor adverse events. The most prevalent adverse events were the common cold ( 2 in the OE group and 4 in the placebo group), being followed by gastrointestinal upset (4 in the placebo group). It is noteworthy that 2 cases in the placebo group but none in the OE group had hangovers. These self-recorded adverse events were mild in intensity, occurred only temporarily and were judged by the investigator as not associated with the treatment. In both study groups, routine laboratory tests (with the exception of GGT) and measurements of anthropometric and hemodynamic parameters did not show any clinically significant abnormalities throughout the intervention.

\section{Discussion}

The present pilot randomized, double-blind, placebo-controlled study was conducted to evaluate the effect of an OE supplement on liver enzymes in individuals with an alcohol drinking habit who had slightly increased GGT activities. The effectiveness of the OE supplement in improving alcohol-deteriorated liver function was shown by the results of an efficacy assessment based on liver enzymes, particularly the GGT results. In the OE group, serum GGT levels were significantly reduced compared with those in the placebo group after 12 weeks of intervention $(\mathrm{P}<0.05)$. 

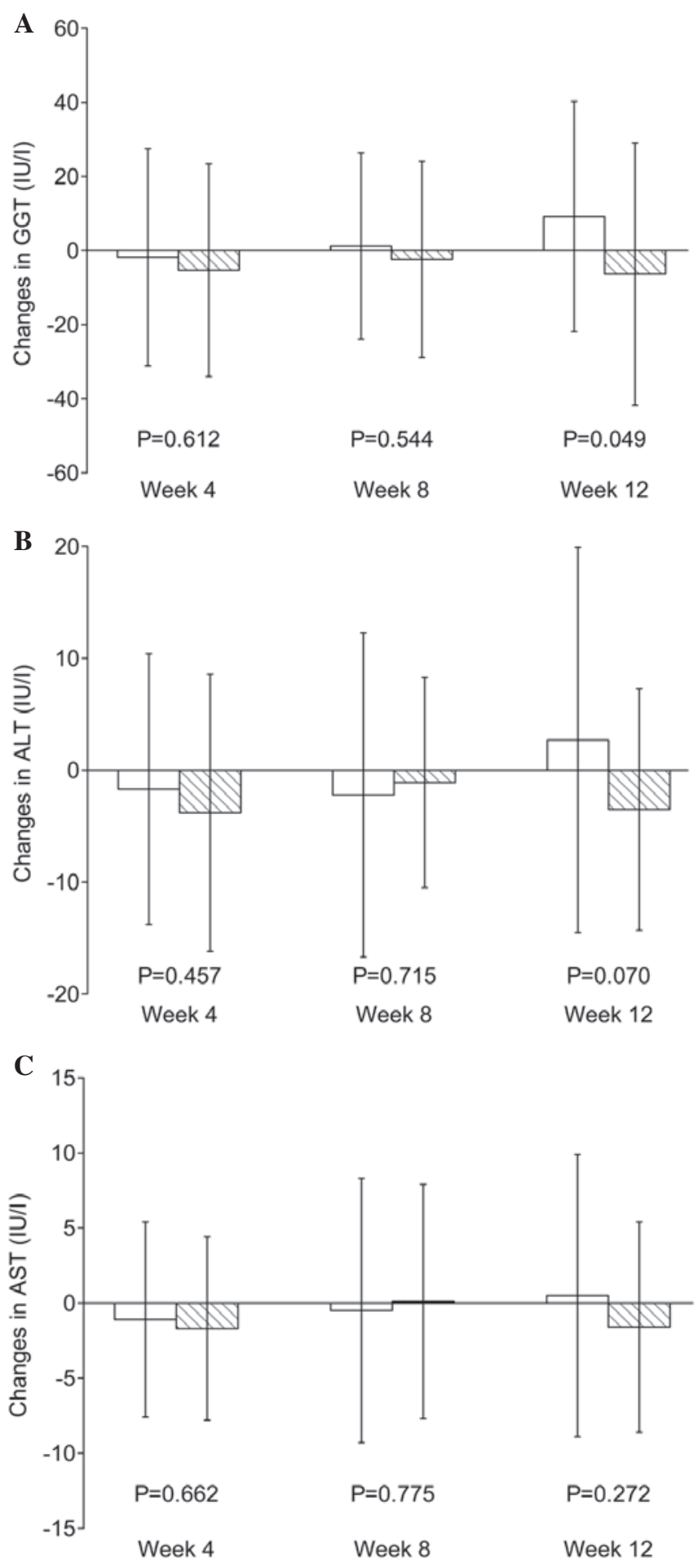

Figure 1. Comparison of changes in serum levels from the value at week 0 of (A) GGT, (B) ALT and (C) AST to the value at weeks 4, 8 and 12 during the 12 week intervention between the placebo group (open bars; $n=36$ ) and the $\mathrm{OE}$ group (diagonally striped bars; $\mathrm{n}=38$ ). Data are presented as the mean \pm standard deviation. The P-value refers to the between-group difference assessed by Student's unpaired t-test. OE, oyster extract; GGT, $\gamma$-glutamyl transferase; ALT, alanine transaminase; AST, aspartate transaminase.

Although ALT and AST levels also appeared to be improved by the OE supplement consumption, there was no significant between-group difference in these two liver enzymes. This can be explained by the fact that baseline mean values of ALT and AST in both groups were within the normal range.
Furthermore, GGT has been found to be the most sensitive and specific marker of alcoholic liver injury and excessive alcohol consumption (17-19,22-24). It is, therefore, considered that the majority of the subjects in the present study were at an early stage of development of ALD, such as hepatic steatosis. Alcoholic steatosis is a reversible disorder, and the reduction of steatosis is reported to arrest the progression of ALD (25). These findings and the data showing that there is no difference between the two groups in the estimated alcohol consumption throughout the study period, indicate that a reduction in GGT by OE supplement is likely to be due to an improvement in alcoholic liver injury.

The efficacy of OE in reducing the serum level of liver enzymes (ALT, AST and/or GGT) has been demonstrated in an animal model of ALD (25), in humans with mild alcoholic liver injury $(26,27)$ and those with chronic hepatitis $(28)$. Consistent with these reports, the present study performed with individuals who had an alcohol drinking habit and a slightly raised GGT showed that OE supplement consumption was effective in decreasing serum GGT levels. Regarding the underlying mechanism of the hepatoprotective action of OE, Ming et al (29) reported that supplementation with OE significantly suppressed the elevation of serum levels of alcohol and acetaldehyde in rats receiving alcohol. This led them to the suggest that $\mathrm{OE}$ is effective in enhancing alcohol metabolism catalyzed by various hepatic enzymes in the alcohol dehydrogenase pathway (29).

The constituents of $\mathrm{OE}$ that are active in hepatoprotection remain to be defined; however, we suggest that taurine may be the major active component for the following reasons: i) The OE preparation for the OE supplement product used in the present study contains a substantial amount of taurine $(>5 \%, \mathrm{w} / \mathrm{w})$; ii) numerous animal studies have demonstrated that hepatic injury due to chronic alcohol administration can be ameliorated by treatment with taurine (13-16,30-32); and iii) there are several other common biological activities, particularly hepatoprotection-associated lipid and anti-oxidative activities, shared by $\mathrm{OE}(33,34)$ and taurine $(6,12)$.

Throughout the 12 -week intervention with the OE supplement at a daily dose of 1,000 $\mathrm{mg}$ (>50 mg as taurine), no untoward side-effects were reported and no abnormalities of any anthropometric parameters or in laboratory test results were observed. The safety of the OE supplement is supported by a previous toxicological study performed with rats showing that there were no significant toxic effects nor any interaction with various therapeutic medicines, such as anti-platelet aggregation drugs (e.g., aspirin) and anti-blood coagulation drugs (e.g., warfarin), following 90-day administration at a daily dose of 1,000 or $2,000 \mathrm{mg} / \mathrm{kg}$ (35). In humans, it is known that the main source of taurine is diet, the amount of daily taurine intake is estimated to range from 40 to $400 \mathrm{mg}$ (36), and that excess taurine is inert and is excreted into the urine unchanged (37).

Based on all the data obtained from this study, it may be concluded that an OE supplement can be safely administered and is effective in improving alcohol-impaired liver function in subjects with an alcohol-drinking habit. Thus, an OE supplement could be a potential candidate for preventing alcoholic liver injury. 


\section{References}

1. Global status report on alcohol and health. World Health Organization, Geneva, Switzerland, 2011.

2. Lu XL, Tao M, Luo JY, Geng Y, Zhao HL and Zhao P: Epidemiology of alcohol and liver disease. Zhonghua Gan Zang Bing Za Zhi 6: 467-468, 2002 (In Chinese).

3. Park SH, Kim CH, Kim DJ, et al: Prevalence of alcoholic liver disease among Korean adults: Results from the Fourth Korea National Health and Nutrition Examination Survey, 2009. Subst Use Misuse 46: 1755-1762, 2011.

4. Kim KA: Current status of liver diseases in Korea: toxic and alcoholic liver diseases. Korean J Hepatol 15 (Suppl 6): S29-S33, 2009.

5. Takada A and Tsutsumi M: National survey of alcoholic liver disease in Japan (1968-91). J Gastroenterol Hepatol 10: 509-516, 1995.

6. Horie Y, Ishii H and Hibi T: National survey of alcoholic liver disease in Japan. Nihon Arukoru Yakubutsu Igakkai Zasshi 39: 505-510, 2004 (In Japanese).

7. Lucey MR, Mathurin P and Morgan TR: Alcoholic hepatitis. N Engl J Med 360: 2758-2769, 2009.

8. Pirmohamed M, Brown C, Owens L, Luke C, Gilmore IT, Breckenridge AM and Park BK: The burden of alcohol misuse on an inner-city general hospital. QJM 93: 291-295, 2000.

9. Pirmohamed $M$ and Gilmore IT: Alcohol abuse and the burden on the NHS - time for action. J R Coll Physicians Lond 34 161-162, 2000.

10. Madhotra R and Gilmore IT: Recent developments in the treatment of alcoholic hepatitis. QJM 96: 391-400, 2003.

11. Barve A, Khan R, Marsano L, Ravindra KV and McClain C: Treatment of alcoholic liver disease. Ann Hepatol 7: 5-15, 2008.

12. Huxtable RJ: Physiological actions of taurine. Physiol Rev 72: 101-163, 1992.

13. Chen X, Sebastian BM, Tang H, McMullen MM, Axhemi A, Jacobsen DW and Nagy LE: Taurine supplementation prevents ethanol-induced decrease in serum adiponectin and reduces hepatic steatosis in rats. Hepatology 49: 1554-1562, 2009.

14. Kerai MD, Waterfield CJ, Kenyon SH, Asker DS and Timbrell JA: Taurine: Protective properties against ethanol-induced hepatic steatosis and lipid peroxidation during chronic ethanol consumption in rats. Amino Acids 15: 53-76, 1998.

15. Wu G, Yang J, Sun C, Luan X, Shi J and Hu J: Effect of taurine on alcoholic liver disease in rats. Amino Acids 36: 457-464, 2009

16. Chang YY, Chou CH, Chiu CH, Yang KT, Lin YL, Weng WL and Chen YC: Preventive effects of taurine on development of hepatic steatosis induced by a high fat/cholesterol dietary habit. J Agric Food Chem 59: 450-457, 2011.

17. Harada S, Misawa S, Agarwal DP and Goedde HW: Liver alcohol dehydrogenase and aldehyde dehydrogenase in the Japanese: Isozyme variation and its possible role in alcohol intoxication. Am J Hum Genet 32: 8-15, 1980.

18. Goedde HW, Agarwal DP, Harada S, Meier-Tackmann D, Ruofu D, Bienzle U, Kroeger A and Hussein L: Population genetic studies on aldehyde dehydrogenase isozyme deficiency and alcohol sensitivity. Am J Hum Genet 35: 769-772, 1983.

19. Matsuda Y, Tsuchishima M, Ueshima Y, Takase S and Takada A: The relationship between the development of alcoholic liver and pancreatic diseases and the induction of gamma glutamyl transferase. Alcohol Alcohol Suppl 1B: 27-33, 1993.

20. Conigrave KM, Davies P, Haber P and Whitfield JB: Traditional markers of excessive alcohol use. Addiction 98 (Suppl 2): 31-43, 2003.
21. Matsuka Y, Wang D-H, Suganuma N, Imai K, Ikeda S, Taketa K and Kira S: Differential responses of serum gamma-glutamyltransferase to alcohol intake in Japanese males. Acta Med Okayama 57: 171-178, 2003

22. Bell H and Steensland H: Serum activity of gamma-glutamyltranspeptidase (GGT) in relation to estimated alcohol consumption and questionnaires in alcohol dependence syndrome. Br J Addict 82: 1021-1026, 1987.

23. Steffensen FH, Sørensen HT, Brock A, Vilstrup H and Lauritzen T: Alcohol consumption and serum liver-derived enzymes in a Danish population aged 30-50 years. Int J Epidemiol 26: 92-99, 1997.

24. Anton RF, Lieber C and Tabakoff B; CDTect Study Group: Carbohydrate-deficient transferrin and gamma-glutamyltransferase for the detection and monitoring of alcohol use: Results from a multisite study. Alcohol Clin Exp Res 26: 1215-1222, 2002.

25. Lakshman MR: Some novel insights into the pathogenesis of alcoholic steatosis. Alcohol 34: 45-48, 2004.

26. Zhang C, Li X, Jing X, Zhang B, Zhang Q, Niu Q, Wang J and Tian Z: Protective effects of oyster extract against hepatic tissue injury in alcoholic liver diseases. J Ocean Univ China 13: 262-270, 2014.

27. Fujisawa H, Takehara I, Nishihori Y, Yano Y, Yamazaki N, Matsui H, Matsuda Y and Fukuhara I: Effect of 12 weeks ingestion of oyster extract on hepatic function in human volunteers with mild alcoholic liver damage. Trace Nutr Res 29: 13-17, 2012 (In Japanese)

28. Matsuda Y, Izuta S, Fujita T, Ohta T, Nakatsuka M, Yoshino M and Shibata Y: Influence of oyster extract administration of diabetes mellitus and hepatitis patients. Trace Nutr Res 12: 91-97, 1995 (In Japanese).

29. Ming Z, Shimizu Y, Furuta K, Tanaka S and Gohda E: Acceleration of alcohol metabolism by Crassostera gigas extract. Jpn Pharmacol Ther 41: 319-321, 2013 (In Japanese).

30. Devi SL, Viswanathan P and Anuradha CV: Regression of liver fibrosis by taurine in rats fed alcohol: Effects on collagen accumulation, selected cytokines and stellate cell activation. Eur J Pharmacol 647: 161-170, 2010.

31. Yang L, Latchoumycandane C, McMullen MR, Pratt BT, Zhang R, Papouchado BG, Nagy LE, Feldstein AE and McIntyre TM: Chronic alcohol exposure increases circulating bioactive oxidized phospholipids. J Biol Chem 285: 22211-22220, 2010.

32. Latchoumycandane C, Nagy LE and McIntyre TM: Chronic ethanol ingestion induces oxidative kidney injury through taurine-inhibitable inflammation. Free Radic Biol Med 69: 403-416, 2014.

33. Tanaka K, Nishizono S, Kugino K, Tamari M, Kurumiya M, Abe N and Ikeda I: Effects of dietary oyster extract on lipid metabolism, blood pressure and blood glucose in SD rats, hypertensive rats and diabetic rats. Biosci Biotechnol Biochem 70: 462-470, 2006.

34. Yoshikawa T, Naito Y, Masui K, Fujii T, Boku Y, Nakagawa S, Yoshida $\mathrm{N}$ and Kondo $\mathrm{M}$ : Free radical-scavenging activity of Crassostera gigas extract (JCOE). Biomed Pharmacother 51: 328-332, 1997.

35. Nishihori Y, Suzuki Y, Kishinami M, Fujisawa H, Nagaoka S, Yamazaki N, Matsui H and Matsuda Y: Study of safety and functionality in oyster extract powder. Trace Nutr Res 28: 40-44, 2011.

36. Wójcik OP, Koenig KL, Zeleniuch-Jacquotte A, Costa M and Chen Y: The potential protective effects of taurine on coronary heart disease. Atherosclerosis 208: 19-25, 2010.

37. Chesney RW: Taurine: Its biological role and clinical implications. Adv Pediatr 32: 1-42, 1985. 